\title{
Evaluación del impacto del emprendimiento empresarial en los estudiantes de la carrera de Administración y Gestión de la Upemor, México
}

\section{Entrepreneurship impact of evaluation of managemente students of the Universidad Politécnica del Estado de Morelos UPEMOR, México}

Universidad Politécnica del Estado de Morelos, Upemor, México. Carrera de Administración y Gestión.

Correo electrónico: Imenez@upemor.edu.mx

\section{Resumen}

En este artículo se presentan los alcances, la metodología y los hallazgos del proyecto Evaluación del impacto del emprendimiento empresarial en los estudiantes de la carrera de administración y gestión de la UPEMOR. Su objetivo fue obtener y conocer las acciones encaminadas a medir el impacto del emprendimiento empresarial de los estudiantes de la carrera de Administración y Gestión de la Universidad Politécnica del Estado de Morelos. Su validez está fundamentada en la carencia de lineamientos verdaderamente efectivos para la medición de los niveles del emprendimiento universitario. Toda la información recabada se analizó con la ayuda de los programas estadísticos SPSS 22.0 y de Excel. Para recolectar la información, se diseñaron y aplicaron cuestionarios, en los que se indagaba sobre características emprendedoras, creación de empresas, capacitación y formación. Se comprobó que la red de contactos empresariales tiene una relación significativa con la decisión del universitario de fundar su propia empresa. Es decir, quedó demostrado que, cuando un alumno conoce personalmente a un emprendedor (sea familiar o no), eso influye en su posible decisión de crear una empresa.

Palabras clave: emprendimiento, estudiantes, instituciones, formación, espíritu empresarial, universidad. 


\section{Abstract}

In this article, are presented the reaches, the methodology and the findings of the titled project "Evaluation of the entrepreneurship's impact in the students of administration career and UPEMOR 's management". Its objective was obtain and know the routed actions to measure the entrepreneurship's impact in the students of administration career and management of the Polytechnic University of the Morelos State. Its validity is substantiated in the lack of guidelines truly effectives for measurement of the university entrepreneurship levels. All the collected information was analyzed with the help of the statistic programs SPSS 22.0 and Excel. To collect the information, they designed and applied questionnaires, in which it was inquired about entrepreneurial characteristics, business creation, training and formation. It was checked that the network of business contacts has a significant relationship with the academic decision of start up his own company. That is to say, it was remain demonstrated when a student knows personally an entrepreneur (be a familiar or not), thats influences his possible decision of create a company.

Keywords: entrepreneurship, students, institutions, training, entrepreneurship, university.

\section{Introducción}

La actitud emprendedora, manifestada durante los últimos 25 años, ha sido una gestión conjunta y pionera de los gobiernos, regiones, universidades, empresas y personas en todos los rincones del planeta; obviamente, con diversos niveles de intensidad. El emprendedurismo es, sin duda alguna, uno de los temas más apasionantes que ocupa el quehacer de muchas personas, la mayoría de ellas, vinculadas al entorno universitario.

La Universidad Politécnica del Estado de Morelos, desde su creación, ha manejado un modelo educativo basado en competencias, con el fin de que los estudiantes desarrollen habilidades, destrezas, aptitudes y conocimientos. Uno de los objetivos primordiales de este modelo educativo es impulsar al alumno a desplegar su espíritu emprendedor, a través de materias como Estadía y Estancia, que per- miten al estudiante tener un contacto más estrecho con la empresa y conocer su funcionamiento, en todos los ámbitos operativos.

Después de doce años de estar operando, no se ha medido el impacto del emprendimiento empresarial en los estudiantes, motivo por el cual se realizó esta investigación. En la primera etapa, se hizo con los estudiantes de la carrera de Administración y Gestión; posteriormente, con las siete carreras restantes. La muestra estuvo representada en 190 alumnos de tercero, sexto y noveno cuatrimestre, del periodo escolar mayo - agosto del 2015; a quienes se les aplicó una encuesta. En esta investigación, se manejaron ocho variables: intención emprendedora, atracción hacia profesiones, valoración social, habilidades específicas, formación y experiencia, contactos empresariales, educación en el espíritu emprendedor y motivos y frenos. Todo encaminado a determinar el impacto del espíritu emprendedor. Se utilizó la estadística multivariada, empleando ANOVA de un factor, para poder medir las variables, y se plantearon tres hipótesis nulas. El resultado general fue que los universitarios encuestados se ven -a sí mismos- bastantes capacitados para desarrollar aquellas habilidades necesarias para la puesta en marcha y mantenimiento de su propia empresa.

En la actualidad, la generación de ideas -entre los estudiantes de la carrera de Administración y Gestión de la UPEMOR- es abundante. Sin embargo, la mayoría de las veces, estas ideas no se realizan; por ende, se les impide a los estudiantes universitarios ser verdaderos emprendedores. Ellos, al generar las ideas, están desarrollando creatividad e ingenio, con miras a obtener beneficios a corto, mediano y largo plazo. La generación de emprendedores universitarios es altamente importante para la Universidad Politécnica del Estado de Morelos, el Estado y el país, debido a los impactos positivos en lo económico, por ejemplo, en la generación de empleo, el aumento del PIB y de inversiones mayores. A pesar de estos beneficios, existen diversas barreras que impiden el desarrollo del espíritu empresarial en una sociedad. En el caso particular de la UPE$\mathrm{MOR}$, hay proyectos emprendedores que no llegan a culminarse o tienen una corta vida, porque las instituciones gubernamentales y no gubernamentales no informan sobre los apoyos financieros, técnicos, capacitación, tutoría y servicios administrativos; lo 
cual representaría un soporte para la consolidación de sus proyectos.

Debido a los altos niveles de desempleo, la escasez y la baja calidad de los empleos existentes, en un país como México, las personas han creado cierta necesidad urgente de generar sus propios recursos, para lograr independencia y estabilidad económica y, de paso, mejorar su calidad de vida. El mundo se ha dado cuenta de que el emprendimiento es uno de los mejores caminos para crecer económicamente como persona, como organización y, en sentido más amplio, como país. Por esto, los gobiernos han creado programas de apoyo para fomentar el emprendimiento, como el Instituto Nacional del Emprendedor.

Este proyecto de investigación fundamenta su validez en la carencia de lineamientos verdaderamente efectivos para la medición de los niveles del emprendimiento universitario. Al respecto, se habla mucho, pero son contados los estudios existentes; inclusive los que deben actualizarse acorde con las políticas de desarrollo regional. Esto es contradictorio con lo que la universidad hace: desarrolla 3 pilares esenciales: la investigación, la extensión y la pedagogía. Estas dos últimas son acciones fundamentales para generar un óptimo impacto en las empresas del entorno. Esta deficiencia en los indicadores de emprendimiento coloca -no solo la educación morelense, sino el país- en un sitial poco deseable, respecto a los temas de investigación y desarrollo que hacen más competitivo el entorno formativo empresarial morelense.

\section{Bases teóricas}

Para emprender la investigación, fue necesario dejar claros los conceptos utilizados. El emprendedurismo, a pesar de que existe desde hace largo tiempo, como objeto de estudio y de investigación, se le considera como un "paradigma joven sin fundamento teórico sustancial" (Bygrave y Hofer, 1991). Otros lo consideran preparadigmático (Rusque, 2004) por cuanto, a pesar de tener larga trayectoria, los estudiosos del tema no tienen claridad aún sobre las principales categorías y conceptos que se deben manejar para que adquieran una validez común. Para no entrar en polémicas, en este estudio se maneja el concepto del Centro de Empren- dedurismo de la Universidad de Miami en Ohio:"el proceso de identificar, desarrollar y dar vida a una visión, que puede ser una idea novedosa, una oportunidad o simplemente una mejor manera de hacer las cosas; y cuyo resultado final es la creación de una nueva empresa, formada bajo condiciones de riesgo y considerable incertidumbre".

Soto (2004) define emprendedor como "aquel que germina ideas y las pone a crecer para después salir a sembrar de nuevo con su creatividad e ingenio. $Y$ las mejores ideas son aquellas que después vuelan solas sin que su inventor tenga que conducirlas toda la vida". Por su parte, Comeche (2004) explica que "el emprendedor es visto como aquel que percibe una oportunidad y que crea una organización para conseguir dicha oportunidad". En este contexto, se puede afirmar que perseguir una oportunidad para crear una organización está implícito en el acto emprendedor.

Otra idea que debe quedar clara, y que parte del acto de emprender, es cómo iniciar el proceso de emprendimiento (Kantis, 2002). Este implica todas las funciones, tareas y actividades asociadas a la percepción de oportunidades y a la creación de organizaciones para perseguirlas.

Cuando se va a emprender la creación de una empresa, el primer paso es convertirse en empresario, pero sin dejar a un lado su característica de emprendedor. Ripollés (1995) manifiesta, en su obra, que el término empresario ha ido evolucionando hasta llegar a relacionarse -en la literatura especializada- con el progreso económico y social de una comunidad. Destaca que, aunque esto es así, se le ha prestado muy poca atención en el marco de la teoría económica. Precisamente, con base en ello, Comeche (2004) define el empresario como "la persona que emprende un negocio, o la que ha creado una empresa en marcha de la nada".

Hablar de creación de empresas está relacionado con el empresario que vislumbra una oportunidad, que anteriormente se decía era de lucro; pero, hoy, es una oportunidad de actuar con responsabilidad moral y social. Así se llega al término emprendedor universitario. Se define como el ser humano, con estudios superiores, que tiene el suficiente conocimiento y autoconocimiento. Que está motivado 
para iniciar y desarrollar sus sueños, de una manera innovadora, asertiva y proactiva; en diferentes escenarios -sectores económicos o sociales- donde le toque gestionar. Puesto que cuenta con las apropiadas competencias que lo hacen un individuo especial, destinado a ser eficiente. Todo esto lo llevará a producir impactos en la rama productiva que lo seduzca.

La educación en emprendimiento ha evolucionado notablemente los últimos años. En Estados Unidos, se incrementó el número de universidades que ofrecían cursos de emprendimiento: de 16, en 1970, pasó a más de 400 en 1995 (Vesper \& Gartner, 1997). Wilson (2004) realizó un estudio, en el que encontró que la educación en emprendimiento -en Europa- ha estado creciendo significativamente en los últimos 5 años, y se espera que la tendencia se mantenga; esto se refleja en la identificación de 70 centros de emprendimiento, aunque este número puede ser mayor.

En el artículo "Teoría de la Intraempresarialidad" (publicado en la página virtual de la UANL) Knight, Cose \& Baumol hacen referencia a que la "literatura económica ha entendido tradicionalmente el emprenderismo como la creación de una empresa que inicia con una jerarquía a cargo del empresario" (Dyer 1994, Westhead \& Wright, 1998). Sin embargo, en la literatura de gestión, hace aproximadamente dos décadas se ha multiplicado en un sinnúmero de estudios, una nueva perspectiva acerca del emprenderismo. Dicen que es aquel proceso que no necesariamente comienza en la cima, sino que puede iniciarse en la base de la escalera jerárquica e ir ascendiendo paulatinamente.

Dicho proceso emprendedor es el proceso intraempresarial (conocido, en el mundo anglosajón, como intrapreneurship, combinación de los vocablos "intra-firm" $y^{\prime \prime}$ entrepreneurship"). Se distingue del concepto tradicional del emprenderismo, básicamente, en que el proceso de innovación (que implica el acto empresarial) se lleva a cabo dentro del contexto de las organizaciones existentes. Existe otra diferencia, en cuanto al entorno del proceso intraempresarial o de intrapreneurship: el que enmarca al tradicional proceso empresarial. El proceso intraempresarial no está sometido a la presión del proceso competitivo, como ocurre con el proceso puramente empresarial, sino que es seleccionado y reconocido dentro de una estructura jerárquica.
Las encuestas pioneras (tanto a investigadores como a empresarios) de Gartner (1990), sobre el tema, muestran que la aplicabilidad de la perspectiva empresarial -como fundador, dueño y gerente- es demasiado elemental. De hecho, no fue clasificada dentro de los temas más importantes que examinaron los expertos en emprenderismo. Cuatro de cinco expertos centraron sus estudios en las características del espíritu emprendedor y solamente uno de cinco se centró en los resultados del espíritu emprendedor. El problema proviene, según lo observado, de una definición demasiado reducida acerca del proceso empresarial y del contexto en el que se ha circunscrito tradicionalmente.

Como ya se ha mencionado, varios estudios han intentado extender la definición del espíritu emprendedor, como la distinción entre los empresarios innovadores, fuera y dentro de las organizaciones existentes (Gartner, 1989; Carlandcet al., 1984). Investigaciones como las de Sharman \& Chrisman (1999) proponen una definición para el emprenderismo que es altamente consistente con los autores principales del emprenderismo (Schumpeter, 1934 y Gartner, 1990) y del proceso intraempresarial, conocido, también, como emprenderismo corporativo (Zahra, 1995). Además, identifican a los empresarios como individuos o grupos de individuos que actúan, ya sea independientemente, o como parte de un sistema corporativo, que crean nuevas organizaciones, o instigan la renovación o innovación dentro de una organización existente (Sharman \& Chrisman, 1999). Esta definición indica que el espíritu emprendedor no deberá limitarse más a la descripción de los esfuerzos emprendedores de los individuos que funcionan solamente fuera del contexto de una organización existente. Además, las actividades emprendedoras, llevadas a cabo independientemente y aquellas emprendidas dentro del contexto una organización, ya pueden distinguirse entre sí, como emprenderismo independiente e intraemprenderismo o intrapreneurship, respectivamente.

El emprenderismo independiente es el proceso por el cual un individuo o un grupo de individuos, actuando independientemente, crea una nueva organización. El intraemprenderismo o intrapreneurship es el proceso por el cual un individuo o grupo de individuos, en asociación con una organización 
existente, crea una nueva organización o instiga la renovación o innovación dentro de la organización (Sharman \& Chrisman, 1999). Una variedad enorme de conceptos describe esfuerzos emprendedores dentro de una organización existente como el emprenderismo corporativo (Burgelman, 1983), el venture corporativo (Biggadike, 1979), emprenderismo corporativo interno (Jones y Butler, 1992), emprenderismo corporativo externo (Schollhammer, 1982; Vesper, 1984) y renovación estratégica (Guth \& Ginsberg, 1990); entre otros.

La característica más interesante -de esta clasificación, para esta investigación- es la presencia de la innovación, como condición suficiente para el emprenderismo; aunque no es una condición necesaria. La creación y/o la renovación de la organización pueden ocurrir en ausencia de la innovación, ya que la innovación, o novedad, es una cuestión de grado. Además, nuevo en el mercado, no significa necesariamente que la innovación esté vendida, como en el caso de una forma de organización. Como la innovación puede variar en su cantidad e impacto, es muy difícil y, de hecho, ineficaz procurar especificar el nivel exacto de la innovación necesaria para el espíritu emprendedor. Por tanto, es mejor entender la innovación como un acto de emprenderismo, más que como el único acto del proceso emprendedor (Sharma \& Chrisman, 1999).

Con base en lo anterior, es posible afirmar que todos los incentivos, conducentes a las innovaciones, realzan el proceso emprendedor; pero no todos los incentivos, conducentes al proceso emprendedor del aumento, lo hacen. Por lo tanto, la tarea de encontrar el ambiente institucional apropiado para el espíritu emprendedor puede no ser suficiente para encontrar el apropiado para la innovación. Así pues, está el dilema de investigar las medidas - para desarrollar el proceso emprendedor- que no aumentan comportamiento innovador, sino que, simplemente, crean o renuevan las organizaciones en las cuales la innovación está ausente. Entonces, hay que centrarse en esta pregunta: ¿qué incentivos institucionales son aquellos que realzan los actos emprendedores relacionados con la innovación? Es un hecho que la promoción de la innovación debería ser una prioridad para las firmas que desean mantener su competitividad con una globalización del mercado en aumento (Carrier, 1996).
Recordemos que el proceso innovador (schumpeteriano) es la introducción de una invención o de una idea original en una forma comercialmente usable, que sea nueva en el mercado y tenga el potencial de transformar el ambiente competitivo, así como la organización (Stopford Baden-Baden-Fuller, 1994). El proceso de la innovación intraempresarial adquiere importancia especial en países como México, porque la mayoría del valor agregado se crea dentro de las firmas existentes. Sin embargo, se verá más adelante, el cambio estructural no ha favorecido las actividades innovadoras. Esto ha aumentado el número de firmas de baja productividad que reducen el nivel promedio total de la productividad de trabajo y el crecimiento industrial. Así, queda evidente que las instituciones intraorganizacionales no han sido las apropiadas para conducir el emprenderismo innovador dentro de la firma.

Crear una nueva oportunidad de generación de recursos está relacionada con la labor de 'emprender', palabra que se deriva del francés entrepende y significa 'llevar a cabo'. Según Jmaes W. Halloran (1997), el emprendedor es aquel que asume el riesgo de obtener beneficios o incurrir en pérdidas realizando operaciones empresariales. El emprendedor es un empresario que lleva a cabo una aventura, la organiza, busca capital para financiarla y asume el riesgo. Esto indica la necesidad de explorar, en el ser humano, la estructura compleja de su personalidad que, de una u otra forma, influye en la orientación hacia el emprendimiento.

\section{Aspectos metodológicos}

En el diagrama de flujo siguiente (figura 1) se muestran los pasos que se deben seguir en el desarrollo de la metodología:

Para recolectar la información, se diseñaron y aplicaron cuestionarios, en los que se indagaba sobre características emprendedoras, creación de empresas, capacitación y formación. El cuestionario se aplicó a un universo de 377 estudiantes de la carrera de Administración y Gestión, y se tomó, como población objetivo, los 190 estudiantes de tercero, sexto y noveno cuatrimestre. 


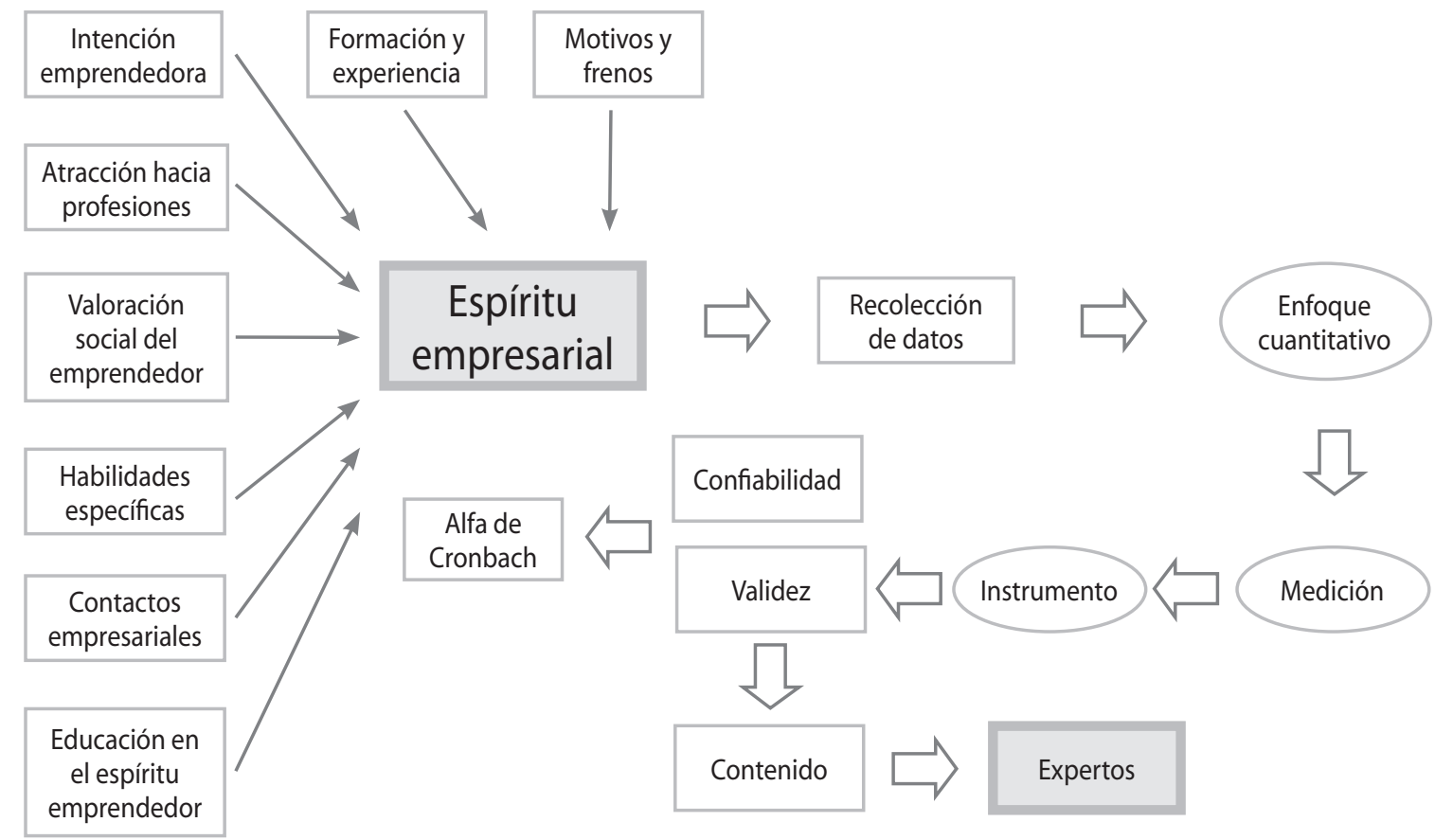

Figura1: Diagrama de Flujo.

Fuente: Elaboración propia

\section{Instrumento de recolección de datos}

Los datos utilizados para la realización del presente estudio, se obtuvieron de la encuesta a los alumnos de la carrera de Administración y Gestión de la Universidad Politécnica del Estado de Morelos. Estas encuestas se hicieron en forma presencial, durante los meses de mayo y junio de 2015, mediante un muestreo, basado en el método de conveniencia y cuotas. Los alumnos respondían el cuestionario en el aula, anónimamente, durante 15 minutos. El número total de encuestas recogidas fue 190. Toda esa información recabada se analizó con la ayuda de los programas estadísticos SPSS 22.0 y Excel (Tabla 1). La encuesta está formada por 9 bloques, a través de los cuales se puede obtener información, que, según la literatura previa estudiada, puede influir en la intención emprendedora de los universitarios (Chen et al. 1998; Krueger, 2000; Kouriloff, 2000; Urbano, 2006; Mira Solves, 2006; Guerrero y Urbano, 2007; Liñán et al. 2008; Lucas et al. 2009; Liñán et al. 2011).

El cuestionario contemplaba estas categorías:

a) Intención emprendedora. Se preguntó acerca de: la dificultad de la creación y mantenimiento de una empresa, la opinión de sus amigos, compa- ñeros y familiares en el caso de que decidiera crear una empresa, así como su propio grado de satisfacción personal en dicho caso.

b) Atracción hacia profesiones. Se cuestionó el grado de atracción que muestra el alumno hacia el trabajo asalariado o el emprendimiento y hacia la profesión independiente. Teniendo en cuenta todas las ventajas y desventajas (económicas, personales, reconocimiento social, seguridad laboral, etc.) que ello conlleva.

c) Valoración social del emprendedor. Aquí, se preguntó sobre el concepto que se tiene del emprendedor, dentro de la familia, los compañeros y la cultura general del país del encuestado.

d) Habilidades específicas para emprender. Estas tienen que ver, por ejemplo, con conocimientos de mercadotecnia, negociación con proveedores, selección de personal o diseño de un plan de negocios; entre otros.

e) Formación y experiencia. Se indagó sobre el nivel de estudios y titulación en la que se encuentra el estudiante, su experiencia laboral, su posible relación con el autoempleo, así como su serio convencimiento de crear una empresa propia.

f) Motivos y frenos para la creación de una empresa propia. Se les plantearon dos bloques de preguntas 
que deben valorar, según crean que son más o menos importantes. Por un lado, los motivos que pueden favorecer la creación de empresas, como: las recompensas económicas, la independencia personal, la seguridad personal o familiar, el reconocimiento o la necesidad de inversión de un patrimonio personal. Por otro lado, los frenos: el riesgo económico, las necesidades de capital inicial, las cargas fiscales, la falta de tiempo para la realización de otras actividades, la falta de conocimientos para poner en marcha una empresa o el temor al fracaso.

g) Contactos empresariales. Se cuestionó acerca de los posibles contactos que los alumnos pueden tener dentro de la familia, amigos, jefes, profesores, como también, la percepción que las personas tienen de ellos, como emprendedores de éxito.

h) Educación en el espíritu emprendedor. Se les preguntó directamente; después, a aquellos que respondieron afirmativamente, debían indicar el grado de acuerdo o desacuerdo, respecto a una serie de actividades que se pueden considerar importantes para incluir en estos cursos relacionados con la creación de empresas.

i) Datos generales. Se les indagó sobre la edad, el sexo, lugar de nacimiento, de residencia, el nivel de estudios y ocupación actual de sus padres. Estos factores han demostrado influir sobre la intención.

\section{Análisis de los resultados}

Antes de analizar los resultados, es importante manejar los siguientes conceptos; en primer lugar, las intenciones. Triandis (1980) y Ajzen (2005) son dos de los autores de referencia que las han estudiado. Para Triandis (1980), "son las instrucciones que las personas se dan a sí mismas para comportarse en ciertas formas". Por su parte, Ajzen (2005) las define como"aquellos comportamientos que las personas pueden ejecutar primordialmente bajo un control voluntario". Con base en esta definición, Ajzen \& Fishbein (1980) establecieron la teoría de la Acción Razonada, que posteriormente sería revisada por ellos mismos en el año 2010. Esta teoría adquirió el nombre de Teoría del Comportamiento Planeado, y establece que la intención se compone de tres determinantes principales: la actitud hacia el comportamiento, la norma subjetiva y el control de comportamiento percibido.

Para evaluar si el alumno ha pensado seriamente, alguna vez en fundar una empresa propia, se le preguntó directamente; las respuestas están consignadas en la Tabla 1. Como puede observarse, un 95.27\% del alumnado ha pensado en algún momento en la creación de su propia empresa; 38.42\% lo ha hecho'seriamente'. Solo un $24.22 \%$ lo ha hecho 'vagamente'. El 32.63\% tiene el firme propósito de llevarlo a cabo y únicamente el $4.73 \%$ no lo haría.

Posteriormente, se aplicó la prueba Chi-cuadrado de Pearson, para comprobar la relación que puede existir entre la variable" pensar seriamente en crear una empresa propia" y algunas de las variables de las que resulta más interesante averiguar si están relacionadas o no. Dichas variables están relacionadas con los estudios, el curso, el género o la experien-

Tabla 1. Pensar seriamente en crear una empresa propia

\begin{tabular}{|c|c|c|c|c|c|}
\hline & & \multirow{3}{*}{$\begin{array}{c}\text { Frecuencia } \\
46\end{array}$} & \multirow{3}{*}{$\begin{array}{c}\text { Porcentaje } \\
24.22\end{array}$} & \multirow{3}{*}{$\begin{array}{c}\begin{array}{c}\text { Porcentaje } \\
\text { Válido }\end{array} \\
24.22 \\
\end{array}$} & \multirow{3}{*}{$\begin{array}{c}\text { Porcentaje } \\
\text { Acumulado } \\
24,22\end{array}$} \\
\hline & & & & & \\
\hline Válidos & Sí, vagamente & & & & \\
\hline & Sí, seriamente & 73 & 38.42 & 38.42 & 62.64 \\
\hline & $\begin{array}{l}\text { Sí, tengo firme el } \\
\text { propósito de crear } \\
\text { una empresa }\end{array}$ & 62 & 32.63 & 32.63 & 95.27 \\
\hline & No & 9 & 4.73 & 4.73 & 100 \\
\hline & Total & 190 & 100 & 100 & \\
\hline
\end{tabular}

Fuente: elaboración propia. 
cia laboral; entre otras. Cabe aclarar que, con este método, el contraste de la hipótesis nula (Ho) se hace con el p valor (significación bilateral), o nivel de error, que permite rechazar la hipótesis, cuando es superior a 0.05, que es el nivel máximo de error que se está dispuesto a asumir. Las hipótesis nulas en este método, plantean la independencia entre las variables escogidas para el análisis en cuestión; es decir, se busca contrastar si existe relación entre las variables analizadas.

En los casos en los que se encontró relación -entre las variables analizadas con la Chi-cuadrado- se procedió a aplicar el Coeficiente Phi yV de Cramer. Son valores comprendidos entre 0 y 1 , que deben ser comparados con el valor 0.6; que sirve para comprobar el grado de relación de las variables estudiadas. Si el valor es superior a 0.6, la relación será fuerte; en caso contrario, será débil. La hipótesis nula para contrastar fue: Ho: las variables estudiadas no tienen relación con la decisión de crear una empresa propia (ver Tabla 2).

A continuación, se muestra un cuadro resumen con todos los datos obtenidos, tras aplicar los contrastes anteriormente mencionados (Tabla 2). De él se desprende que son cinco las variables que tienen una relación significativa con el hecho de pensar en crear una empresa propia. Esas variables son: el cur- so, la experiencia laboral, tener algún familiar empresario, tener alguna relación con el autoempleo y conocer personalmente a algún emprendedor. Parece evidenciarse que, para estos estudiantes, conocer a algún emprendedor, tener experiencia profesional o relación con el autoempleo (haber estado autoempleado o haber participado en la creación de alguna empresa) puede influir en la intención de un individuo de fundar su propia empresa. Aunque cabe decir que, aplicando el coeficiente de Phi y V de Cramer, se ve que dicha relación es débil, puesto que el p_valor es inferior a 0.6.

Para el estudio del constructo de intención emprendedora, se recurrió al análisis ANOVA de un factor. Este contraste sirve para saber si la intención de crear una empresa propia está relacionada con los ítems considerados. Este análisis se utiliza para contrastar la hipótesis de que varias medias son iguales.

La hipótesis nula para contrastar fue: Ho: Las creencias respecto al emprendimiento (la actitud personal, la norma subjetiva y el control del comportamiento percibido) no están relacionadas con la decisión de crear una empresa propia.

Puesto que el p_valor de todas ellas es inferior a 0.05 , se rechazó la hipótesis nula planteada. Por lo tanto, se puede afirmar que todas esas afirmaciones

Tabla 2. Chi-cuadrado de Pearson

\begin{tabular}{|c|c|c|c|c|}
\hline & \multirow[t]{2}{*}{ Variables } & \multicolumn{2}{|c|}{ Chi cuadrado de Pearson } & oeficiente Phi y V de Cramer \\
\hline & & P_valor & Aceptar/Rechazar Ho & Grado de relación \\
\hline & Estudios & 0.426 & Aceptar & \\
\hline Pensar & Curso & 0.03 & Rechazar & Débil \\
\hline seriamente & Género & 0.457 & Aceptar & \\
\hline en crear & Experiencia laboral & 0.031 & Rechazar & Débil \\
\hline una & Familiar emprendedor & 0.000 & Rechazar & Débil \\
\hline empresa & Estudios padre & 0.222 & Aceptar & \\
\hline propia & Estudios madre & 0.964 & Aceptar & \\
\hline & Profesión padre & 0.103 & Aceptar & \\
\hline & Profesión madre & 0.86 & Aceptar & \\
\hline & Autoempleo & 0.033 & Rechazar & Débil \\
\hline & $\begin{array}{l}\text { Conocer algún } \\
\text { emprendedor }\end{array}$ & 0.000 & Rechazar & Débil \\
\hline
\end{tabular}

Fuente: elaboración propia. 
contenidas en dicho constructo influyen en el estudiante a la hora de decidir si crear una empresa, o no. En la Tabla 3, se muestra el resumen de dicho contraste. Una vez comprobada la significación de cada una de las variables, el siguiente paso fue probar las medias de cada uno de los ítems. En el mismo cuadro, se puede apreciar, globalmente que los tres ítems que tienen una valoración media más elevada son: Totalmente incapaz de crear una empresa, crear una empresa y mantenerla es fácil y entre varias opciones preferiría ser cualquier cosa antes que emprendedor.

La información más interesante de la Tabla 3 es la de los datos concernientes a los estudiantes que tienen el firme propósito de crear una empresa y a aquellos que no lo han pensado. En relación con los primeros, cabe destacar que dan una mayor valoración media a las variables que hacen referencia a la satisfacción que les supondría ser empresarios, al hecho de tener la oportunidad y los recursos necesarios para crear la empresa y a la aprobación de su actitud por parte de los amigos. Los universitarios que no han pensado en crear una empresa dieron una valoración más elevada a la aprobación de su decisión por parte de amigos, familia y compañeros.

En la Tabla 4, se observa que la profesión que presenta una mayor valoración (4.0073) es la de empresario. Aunque se puede ver que las otras dos opciones también tienen una alta valoración. Este dato resulta llamativo, si se tiene en cuenta la infor-

Tabla 3. Valoraciones medias de intención emprendedora-pensar seriamente en crear una empresa

\begin{tabular}{|c|c|c|c|c|c|c|}
\hline \multicolumn{7}{|c|}{ Medias } \\
\hline Variables & $\begin{array}{l}\text { Sí vagamente } \\
\text { (54) }\end{array}$ & $\begin{array}{c}\text { Sí } \\
\text { seriamente } \\
(68)\end{array}$ & $\begin{array}{l}\text { Sí tengo el firme } \\
\text { propósito de crear } \\
\text { una empresa (58) }\end{array}$ & №. (10) & $\begin{array}{l}\text { Media total } \\
\quad(190)\end{array}$ & sig. \\
\hline Crear una empresa y mantenerla es fácil & 3.07 & 3.32 & 3.26 & 3.60 & 3.25 & 0.393 \\
\hline $\begin{array}{l}\text { Muchas personas consideran poco } \\
\text { aceptable ser emprendedor }\end{array}$ & 2.06 & 2.12 & 1.95 & 2.60 & 2.07 & 0.43 \\
\hline $\begin{array}{l}\text { Amigos aprueban decisión de crear } \\
\text { empresa }\end{array}$ & 1.72 & 1.81 & 2.02 & 1.70 & 1.84 & 0.248 \\
\hline Totalmente incapaz de crear una empresa & 3.44 & 3.9 & 3.74 & 3.20 & 3.58 & 0.009 \\
\hline $\begin{array}{l}\text { Familia aprobaría decisión de crear una } \\
\text { empresa }\end{array}$ & 2.04 & 2.1 & 2.36 & 2.20 & 2.17 & 0.007 \\
\hline $\begin{array}{l}\text { Si tuviese oportunidad y recursos crearía } \\
\text { una empresa }\end{array}$ & 2.19 & 2.53 & 2.66 & 2.40 & 2.45 & 0.000 \\
\hline $\begin{array}{l}\text { Compañeros aprobarían decisión de crear } \\
\text { una empresa }\end{array}$ & 1.96 & 1.78 & 2.14 & 1.90 & 1.95 & 0.075 \\
\hline $\begin{array}{l}\text { Entre varias opciones preferiría ser } \\
\text { cualquier cosa antes que emprendedor }\end{array}$ & 3.06 & 3.26 & 3.48 & 2.90 & 3.25 & 0.228 \\
\hline $\begin{array}{l}\text { Si tratase de ser una empresa tendría } \\
\text { una alta probabilidad de lograrlo }\end{array}$ & 1.89 & 2.04 & 2.57 & 2.30 & 2.17 & 0.000 \\
\hline $\begin{array}{l}\text { Ser empresario me suprondría una gran } \\
\text { satisfacción }\end{array}$ & 1.98 & 2.25 & 2.50 & 2.10 & 2.24 & 0.000 \\
\hline $\begin{array}{l}\text { Muy difíil sacar adelante un proyecto } \\
\text { emprendedor }\end{array}$ & 2.05 & 2.24 & 2.16 & 1.80 & 2.14 & 0.616 \\
\hline $\begin{array}{l}\text { Ser empresario me reportaría más } \\
\text { ventajas que incovenientes }\end{array}$ & 1.87 & 2.09 & 2.36 & 2.10 & 2.11 & 0.014 \\
\hline $\begin{array}{l}\text { Conozco los detalles prácticos necesarios } \\
\text { para crear una empresa }\end{array}$ & 1.72 & 1.96 & 2.22 & 2.30 & 1.99 & 0.015 \\
\hline
\end{tabular}

Fuente: elaboración propia. 
Tabla 4. Descriptivos atracción hacia diferentes profesiones

\begin{tabular}{|l|c|c|c|c|c|}
\hline \multicolumn{1}{|c|}{} & N & Mínimo & Máximo & Media & Desv. Típ \\
\hline Trabajo asalariado & 190 & 1,00 & 5,00 & 37,920 & 101,841 \\
\hline Profesional independiente & 190 & 1,00 & 5,00 & 35,730 & 105,358 \\
\hline Empresario & 190 & 1,00 & 5,00 & 40,073 & 102,708 \\
\hline N válido (según lista) & 190 & & & & \\
\hline
\end{tabular}

Fuente: elaboración propia.

mación (dada en párrafos anteriores), que decía que solo un $32.63 \%$ de los encuestados tiene el firme propósito de crear una empresa.

Otro constructo, según Liñán et al. (2011), y Guerrero (2007), es la valoración social que se tiene del emprendedor. Para analizarlo, se realizó un análisis ANOVA. Se contrastó un factor entre los distintos ítems que componen el constructo y lo relacionado con pensar en crear una empresa. Así se comprobó si estas variables están relacionadas con la intención de crear una empresa. Los datos obtenidos se exponen en la Tabla 5, en el que se aprecia que son los ítems 1, 4, 5, 6 y 7, los que influyen en dicha intención: la opinión que la familia, los amigos y los compañeros tienen del emprendedor influye en el estudiante, en su decisión de crear una empresa.

$\mathrm{Al}$ igual que en el caso anterior, era importante saber qué es lo que más tienen en cuenta los alumnos que piensan firmemente en crear su propio negocio y los que no. En la Tabla 5, se aprecia cómo aquellos

Tabla 5. Valoraciones medias de la imagen social del emprendedor: pensar seriamente en crear una empresa

\begin{tabular}{|c|c|c|c|c|c|c|}
\hline \multicolumn{7}{|c|}{ Medias } \\
\hline Variables & № (116) & $\begin{array}{c}\text { Sí } \\
\text { vagamente } \\
(16)\end{array}$ & $\begin{array}{c}\text { Sí } \\
\text { seriamente } \\
(37)\end{array}$ & $\begin{array}{l}\text { Sí, tengo el firme } \\
\text { propósito de crear } \\
\text { una empresa (21) }\end{array}$ & $\begin{array}{l}\text { Media total } \\
\text { (190) }\end{array}$ & Sig \\
\hline $\begin{array}{l}\text { Familia valora profesión de } \\
\text { emprendedor por encima de } \\
\text { otras profesiones }\end{array}$ & 1.93 & 2.00 & 2.24 & 2.00 & 2.01 & 0.442 \\
\hline $\begin{array}{l}\text { Cultura de mi país es muy } \\
\text { favorable hacia la actividad } \\
\text { emprendedora }\end{array}$ & 2.56 & 2.19 & 2.14 & 2.00 & 2.38 & 0.147 \\
\hline $\begin{array}{l}\text { El papel del emprendedor en } \\
\text { la economía es poco reconocido }\end{array}$ & 2.20 & 1.56 & 1.92 & 1.86 & 2.05 & 0.141 \\
\hline $\begin{array}{l}\text { Muchas personas consideran } \\
\text { poco aceptable ser emprendedor }\end{array}$ & 2.23 & 2.13 & 1.62 & 1.95 & 2.07 & 0.048 \\
\hline $\begin{array}{l}\text { Se consideran que la actividad } \\
\text { emprendedora merece la pena, } \\
\text { a pesar de los riesgos }\end{array}$ & 2.11 & 2.19 & 2.41 & 2.05 & 2.17 & 0.138 \\
\hline $\begin{array}{l}\text { Compañeros valoran la } \\
\text { actividad emprendedora por } \\
\text { encima de otras profesiones }\end{array}$ & 2.17 & 2.50 & 2.00 & 2.57 & 2.21 & 0.236 \\
\hline $\begin{array}{l}\text { Se tiende a pensar que los } \\
\text { emprendedores se aprovechan } \\
\text { de los demás }\end{array}$ & 3.28 & 2.75 & 2.89 & 3.71 & 3.21 & 0.036 \\
\hline
\end{tabular}

Fuente: elaboración propia. 


\begin{tabular}{|c|c|c|}
\hline & $\begin{array}{c}\text { Media } \\
\mathrm{N}=190\end{array}$ & $\begin{array}{l}\text { Desviación } \\
\text { típica }\end{array}$ \\
\hline $\begin{array}{l}\text { Seleccionar el mejor enfoque de mercadotecnia para introducir en } \\
\text { el mercado un nuevo producto o servicio }\end{array}$ & 3.2226 & 0.85468 \\
\hline Negociar con un proveedor mejores precios para asegurar el éxito de la nueva empresa & 3.5912 & 0.83473 \\
\hline Estimar correctamente los costos de gestión de un nuevo producto o empresa & 3.5912 & 0.83473 \\
\hline $\begin{array}{l}\text { Reconocer potencial de una idea u oportunidad, que permita el surgimiento de } \\
\text { una empresa de nueva creación de forma exitosa }\end{array}$ & 3.5949 & 0.90184 \\
\hline Reclutar a los empleados idóneos para un nuevo proyecto o empresa & 3.6131 & 0.93955 \\
\hline Convecer a un cliente de probar un nuevo producto o iniciativa & 3.5547 & 0.90921 \\
\hline Escribir un plan de negocios de forma clara y completa & 3.3394 & 0.90468 \\
\hline $\begin{array}{l}\text { Trabajar productivamente bajo estrés continuo, presión y conflicto y tomar decisiones } \\
\text { bajo incertidumbre y riesgo }\end{array}$ & 3.1898 & 1.15173 \\
\hline Convertir un avance científico exitoso en una aplicación práctica & 3.1131 & 0.95214 \\
\hline $\begin{array}{l}\text { Desarrollar una investigación e hipótesis científicas originales, así como un plan } \\
\text { para probarlas }\end{array}$ & 2.9745 & 0.97744 \\
\hline $\begin{array}{l}\text { Diseñar y construir algo innovador que funcione de forma muy parecida a } \\
\text { las especificaciones de diseño }\end{array}$ & 3.0912 & 0.98843 \\
\hline
\end{tabular}

Fuente: elaboración propia.

estudiantes que querían montar su propia empresa tienen muy presentes los ítems 1 y 6 . Es decir, si la sociedad considera que la actividad emprendedora merece la pena, a pesar de los riesgos y la opinión que la familia tiene sobre la profesión de emprendedor. Los que no han pensado en crear su negocio valoran, en mayor medida, el ítem 3, que hace referencia al poco reconocimiento que la sociedad tiene hacia el papel del emprendedor. En relación con los datos globales, hay que destacar que, nuevamente, la variable más valorada es la percepción de que la actividad merece la pena, a pesar de los riesgos.

Con el fin de estudiar la percepción que el alumno tiene de sus propias capacidades concretas relacionadas con el emprendimiento (Lucas et al. 2009), se les planteó una batería de cuestiones que debían valorar en una escala de 1 (nada capaz) a 4 (completamente capaz). En la Tabla 6, puede apreciarse que, en las habilidades relacionadas con la selección del personal (con una media de 3.61), es en lo que más capaces se perciben los estudiantes. La media más baja, 2.97, corresponde a las habilidades para desarrollar una investigación científica original, debido probablemente a la titulación objeto de estudio. Cabe resaltar que, aun así, se consideran, en general, bastante capacitados para desarrollar las habilidades que, en el cuestionario, se le plantearon, ya que todos están por encima de la media de la escala.

Posteriormente, se realizó otro análisis ANOVA de un factor. En esta ocasión, la hipótesis nula a contrastar fue: Ho: las habilidades específicas no están relacionadas con la decisión de crear una empresa propia. Puesto que el p_valor, de todas ellas, es inferior a 0.05 , se rechazó la hipótesis nula planteada. Por lo tanto, se puede afirmar que todas esas habilidades están relacionadas con la decisión del estudiante de crear una empresa, o no. En la Tabla 7, se muestra el resumen de dicho contraste. En la Tabla 8, se ob- 
Tabla 7. Valoraciones medias de las capacidades específicas - pensar seriamente en crear una empresa

\begin{tabular}{|c|c|c|c|c|c|c|}
\hline \multicolumn{7}{|c|}{ Medias } \\
\hline Variables & $\begin{array}{l}\text { No } \\
(9)\end{array}$ & $\begin{array}{l}\text { Sí } \\
\text { vagamente } \\
(46)\end{array}$ & $\begin{array}{c}\text { Sí } \\
\text { seriamente } \\
(73)\end{array}$ & $\begin{array}{l}\text { Sí, tengo el firme } \\
\text { propósito de crear } \\
\text { una empresa (62) }\end{array}$ & $\begin{array}{l}\text { Media total } \\
\quad(190)\end{array}$ & Sig \\
\hline $\begin{array}{l}\text { Seleccionar el mejor enfoque de mercadotecnia para } \\
\text { introducir en el mercado un nuevo producto o servicio }\end{array}$ & 3.4167 & 3.4043 & 3.2800 & 2.8000 & 3.2226 & 0.000 \\
\hline $\begin{array}{l}\text { Negociar con un proveedor mejores precios para asegurar } \\
\text { el éxito de la nueva empresa }\end{array}$ & 3.9167 & 3.8868 & 3.5440 & 3.2333 & 3.5912 & 0.000 \\
\hline $\begin{array}{l}\text { Estimar correctamente los costos de gestión de un nuevo } \\
\text { producto } 0 \text { empresa }\end{array}$ & 3.5556 & 3.8491 & 3.3840 & 2.9500 & 3.4015 & 0.000 \\
\hline $\begin{array}{l}\text { Reconocer potencial de una idea u oportunidad, que } \\
\text { permita el surgimiento de una empresa de nueva creación } \\
\text { de forma exitosa }\end{array}$ & 3.8333 & 4.0566 & 3.5920 & 3.0500 & 3.5949 & 0.000 \\
\hline $\begin{array}{l}\text { Reclutar a los empleados idóneos para un nuevo } \\
\text { proyecto o empresa }\end{array}$ & 3.8611 & 3.9434 & 3.6000 & 3.2000 & 3.6131 & 0.000 \\
\hline $\begin{array}{l}\text { Convecer a un cliente de probar un nuevo producto } \\
\text { o iniciativa }\end{array}$ & 3.7778 & 3.9623 & 3.5520 & 3.0667 & 3.5547 & 0.000 \\
\hline Escribir un plan de negocios de forma clara y completa & 3.6667 & 3.6981 & 3.2880 & 2.9333 & 3.3394 & 0.000 \\
\hline $\begin{array}{l}\text { Trabajar productivamente bajo estrés continuo, presión } \\
\text { y conflicto y tomar decisiones bajo incertidumbre y riesgo }\end{array}$ & 3.3333 & 3.5472 & 3.1600 & 2.8500 & 3.1898 & 0.021 \\
\hline $\begin{array}{l}\text { Convertir un avance cientifico exitoso en una aplicación } \\
\text { práctica }\end{array}$ & 3.1667 & 3.3208 & 3.1680 & 2.7833 & 3.1131 & 0.022 \\
\hline $\begin{array}{l}\text { Desarrollar una investigación e hipótesis científicas } \\
\text { originales, así como un plan para aprobarlas }\end{array}$ & 3.0278 & 3.1698 & 3.0160 & 2.6833 & 2.9745 & 0.048 \\
\hline $\begin{array}{l}\text { Diseñar y construir algo innovador que funcione } \\
\text { de forma muy parecida a las especificaciones de diseño }\end{array}$ & 3.3611 & 3.4340 & 3.0240 & 2.7667 & 3.0912 & 0.000 \\
\hline
\end{tabular}

Fuente: elaboración propia.

serva que tanto los estudiantes que quieren fundar su propia empresa como aquellos que no muestran interés en ello, las capacidades a las que les dan una mayor valoración son la selección de personal y la negociación con proveedores. De manera global, la habilidad más destacada es la selección de personal.

Finalmente, siguiendo a Urbano (2006) y Krueger (2000), se procedió a evaluar los motivos y los frenos que pueden condicionar a los universitarios, a la hora de crear su propia empresa. Para ello, se les pidió que valoraran una serie de variables, en una escala de Likert de 1 (nada importante) a 5 (muy importante). Esto puede verse en la Tabla 8. En dicha tabla, se observa que lo que más motiva a la creación de una empresa propia (con una media de 4.09) son las recompensas económicas; seguidas de la independencia, con una media de 4.05. En relación con los frenos, vemos que el riesgo económico es el más valorado (media de 4.01), así como la falta de capital inicial (3.88). 
Tabla 8. Motivos y frenos para crear una empresa

\begin{tabular}{|c|c|c|c|}
\hline & & $\begin{array}{c}\text { Media } \\
\mathrm{N}=190\end{array}$ & $\begin{array}{l}\text { Desviación } \\
\text { típica }\end{array}$ \\
\hline \multirow{5}{*}{ 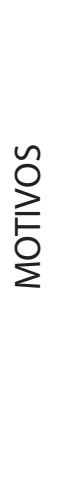 } & Recompensas económicas (incrementar ingresos, etc.) & 4.0912 & 0.86172 \\
\hline & Independencia/autonomía (libertad personal, ser tu propio jefe, etc.) & 4.0511 & 0.88803 \\
\hline & $\begin{array}{l}\text { Recompensas personales (reconocimiento público, crecimiento } \\
\text { personal, probar que soy capaz de hacerlo, etc.) }\end{array}$ & 3.9161 & 0.98164 \\
\hline & $\begin{array}{l}\text { Seguridad familiar y personal (asegurar mi futuro y el de mi familia, } \\
\text { tradición familiar, etc.) }\end{array}$ & 3.8759 & 0.98669 \\
\hline & Invertir y conseguir un patrimonio personal & 4.0000 & 0.87706 \\
\hline \multirow{6}{*}{ 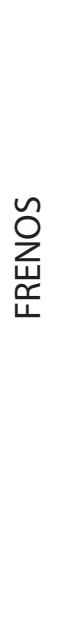 } & $\begin{array}{l}\text { Riesgo económico (riesgo demasiado elevado, situación económica, } \\
\text { falta de un sueldo mínimo asegurado, etc.) }\end{array}$ & 4.0073 & 0.94537 \\
\hline & $\begin{array}{l}\text { Tiempo ilimitado para otras actividades, por tener que trabajar } \\
\text { demasiadas horas (tiempo para la familia, pareja, etc.) }\end{array}$ & 3.4672 & 1.0239 \\
\hline & Temor a fracasar y quedar en ridículo & 3.2117 & 1.23608 \\
\hline & Reparos financieros o de capital (falta de capital inicial) & 3.8796 & 0.9703 \\
\hline & Cargas fiscales & 3.4051 & 0.96843 \\
\hline & $\begin{array}{l}\text { Desconocimiento sobre las regulaciones para poner en marcha } \\
\text { una empresa }\end{array}$ & 3.3394 & 1.12487 \\
\hline
\end{tabular}

Fuente: elaboración propia.

Como se mencionó en la introducción, para Guerrero (2007), resulta especialmente importante saber la educación que han recibido los alumnos en lo que a emprendimiento se refiere. Para ello, se les preguntó si en habían realizado alguna asignatura relacionada con la creación empresas (Tabla 9). En esta ocasión, 81 alumnos respondieron que sí. Posteriormente, se les plantearon dos bloques de cuestiones que debían valorar con una escala Likert. El primer bloque hacía referencia a lo que habían mejorado sus competencias, una vez realizado el curso en cuestión. Y el segundo bloque se refería a las actividades que consideraban importantes en los contenidos de un curso de emprendimiento. Ambos bloques pueden verse en la Tabla 9. En ella, además, puede apreciarse que en lo que más creen mejorar los alumnos, una vez que han hecho los cursos, es en lo referente al conocimiento empresarial, en general. Y en cuanto a las actividades que consideran importantes que se lleven a cabo en dichos cursos, destacan la introducción en situaciones reales. 
Tabla 9. Descriptivos educación en espíritu emprendedor

\begin{tabular}{|c|c|c|}
\hline & $\begin{array}{l}\text { Media } \\
\mathrm{N}=81\end{array}$ & $\begin{array}{l}\text { Desviación } \\
\text { típica }\end{array}$ \\
\hline Conocimiento empresarial & 3.5679 & 0.97389 \\
\hline Mayor reconocimiento de la figura del emprendedor & 3.4568 & 0.92262 \\
\hline Preferencia por ser emprendedor & 3.2716 & 0.8946 \\
\hline Capacidades/habilidades necesarias para ser emprendedor & 3.4815 & 0.9761 \\
\hline Intención/decisión de convertirse en emprendedor & 3.1481 & 0.90982 \\
\hline Promoción del espíritu emprendedor & 3.3333 & 0.98742 \\
\hline Identificación con los emprendedores & 3.2222 & 0.94868 \\
\hline Realización de lecturas y trabajos más que exámenes formales & 3.3827 & 1.00707 \\
\hline $\begin{array}{l}\text { Introducción en situaciones reales (casos de estudio, respuesta a } \\
\text { cambios del enterno, análisis de mercados, etc.) }\end{array}$ & 3.963 & 0.94133 \\
\hline Participación en proyectos con mentores o guías & 3.7778 & 0.93541 \\
\hline Enriquecimiento de las cualidades y capacidades personales & 3.7284 & 0.85165 \\
\hline Elaboración de un plan de negocios & 3.8272 & 0.93261 \\
\hline Contacto o prácticas con expertos 0 emprendedores de la localidad & 3.8642 & 1.04586 \\
\hline $\begin{array}{l}\text { Participación en proyectos, programas o premios existentes hacia } \\
\text { jóvenes emprendedores }\end{array}$ & 3.6049 & 1.03294 \\
\hline Visitas guiadas a empresas, organismos u otras asociaciones & 3.4444 & 1.11803 \\
\hline $\begin{array}{l}\text { Asistencia a congresos o charlas motivacionales donde se prensenta la información de las distintas } \\
\text { prosibilidades de ayuda al emprendedor y la puesta en marcha de nuevas empresas }\end{array}$ & 3.5556 & 1.03682 \\
\hline
\end{tabular}

Fuente: elaboración propia.

\section{Conclusiones y Limitaciones}

Teniendo en cuenta que alrededor de un $38.42 \%$ de los estudiantes encuestados pensó alguna vez en crear su propia empresa, que el $24.21 \%$ lo ha hecho vagamente y que solo el $32.63 \%$ tiene el firme propósito de llevarlo a cabo, es posible concluir lo siguiente:

- Variables como el género, la edad, o los estudios y ocupación de los progenitores no influyen en la decisión de creación de una empresa por parte de los estudiantes.

- Los alumnos que tienen el firme propósito de crear su propia empresa, le dan una valoración mayor a la satisfacción personal que les supondría ser empresarios, al hecho de tener la oportunidad y los recursos necesarios para llevar a cabo sus ideas de negocio y a la aprobación de su actitud, por parte de sus amigos. En cambio, los universitarios que no han pensado en crear su propio negocio le dan una valoración más elevada a la aprobación de su decisión por parte de amigos, familia y compañeros.

- La profesión de empresario es la opción más valorada.

- La imagen que tiene la sociedad del emprendedor influye en la decisión del estudiante, de crear una empresa. De hecho, lo que más influencia tiene en él es la opinión de la familia, amigos y compañeros.

- En general, los universitarios encuestados se ven -a sí mismos- bastante capacitados para desarrollar aquellas habilidades necesarias para la puesta 
en marcha y mantenimiento de su propia empresa. En concreto, en lo que más capaces se muestran es en lo que a selección de personal se refiere.

- En lo que a formación y experiencia respecta, se encontraron relaciones significativas entre el hecho de pensar seriamente en crear una empresa y el cuatrimestre en que se encuentra el alumno, la experiencia profesional y su relación con el autoempleo.

- Los motivos y frenos que más pueden condicionar la intención emprendedora del alumnado son las recompensas económicas y el riesgo económico. Este último, además, tiene una relación significativa, aunque débil, con la decisión de crear una empresa.

- Se comprobó que la red de contactos empresariales tiene una relación significativa con la decisión del universitario de fundar su propia empresa. Es decir, se demuestra que, cuando un alumno conoce personalmente a un emprendedor (sea familiar o no), eso le influye en su posible decisión de crear una empresa.

- Los alumnos que han participado en algún curso relacionado con el emprendimiento, dicen que mejoran sus conocimientos generales empresariales. Además, sugieren que este tipo de cursos deben hacerse en situaciones reales de la vida empresarial.

- La mayor limitación de este estudio fue el hecho de que la encuesta solo se realizó en la carrera de Administración y Gestión de la Universidad Politécnica del Estado de Morelos. Aunque pueda parecer una limitación importante, lo cierto es que de aquí se pueden derivar dos futuras líneas de investigación. La primera de ellas, y se está llevando a cabo: ampliar este estudio al resto de las carreras de la UPEMOR. De este modo, podrían hacerse comparaciones entre las diferentes carreras ofertadas por dicha universidad. Y la segunda, es repetir este mismo estudio en años sucesivos, es decir, realizar un estudio longitudinal de un grupo de estudiantes, desde que comienzan sus estudios universitarios hasta que los finalizan.

\section{Referencias}

Allport, G. W. (1935). Attitudes. In: Murchison, C. (Ed.): Handbook of social psychology. Worcester, Mass. H. Milford, Oxford University Press.
Clark University Press. London, England. http:// web.comhem.se/u52239948/08/allport35.pdf.

Comeche-Martinez, J. M. (2004). Una visión dinámica sobre el emprendedurismo colectivo. Revista de Negocios, 9(2), 1055-1076. http://dx.doi.org/10 $.7867 / 1980-4431.2004 v 9 n 2 p \% 25 p$

Cooper, A. C. \& Dunkelberg, W. C. (1987). Entrepreneurial research: old questions, new answers and methodological issues. Am J Small Bus, 11(3), 11-21.

Cuervo-García, A. (2001). Introducción a la Administración de Empresas ( $6^{\mathrm{a}}$ Ed). Thomson-Civitas Ed. Madrid, España. 454 p.

Crant, J. M. (1996). The proactive personality scale as a predictor of entrepreneurial intentions. J Small Bus Manage, 34(3), 42-49

Davidsson, P. (1995). Culture, structure and regional levels of entrepreneurship. Entrep Region Dev, 7(1), 41-62. http://dx.doi.org/ 10.1080/08985629500000003.

Espíritu-Olmos, R. \& Sastre-Castillo, M.A. (2007). La actitud emprendedora durante la vida académica de los estudiantes universitarios. Cuadernos de Estudios Empresariales, 17, 95-116. http:// revistas.ucm.es/index.php/CESE/article/view/CESE0707110095A/9419

Gallo-Laguna de Rins, M.A. (2003). Preservando el espíritu emprendedor en la empresa familiar: estructuras, sistemas de dirección y órganos de gobierno que facilitan la iniciativa emprendedora. Iniciativa Emprendedora y Empresa Familiar, 39, 7-20.

González-Morales, M.O. (2001). Actitudes y motivaciones hacia el trabajo y la creación de empresas según género. Boletín Económico de ICE, 2709, 21-29.

Guerrero-Cano, M \& Urbano-Pulido, D. (2007). Entrepreneurial Universities: The case of Autonomous University of Barcelona. Research Work. Universidad Autónoma de Barcelona (Eds.). 166 p.

Kantis, H., Ishida, M., \& Komori, M. (2002). Empresarialidad en economías emergentes: creación y desarrollo de nuevas empresas en América Latina y el Este de Asia. En: El emprendedor innovador y la creación de empresas de I+D+I, 14. Servei de Publicacións Universitat de Valencia, Spain. Roig S., Ribeiro D.S, Torcal R., De la Torre A., Cerver E. (Eds.) pp. 239-256.

Krueger, N. (2000). The cognitive infrastructure of opportunity emergence. Entrep Theory Pract, 
24(3), 5-23. http://dx.doi.org/10.1007/978-3-54048543-8_9

Krueger, N.F. \& Brazeal, D.V. (1994). Entrepreneurial potential and potential entrepreneurs. Entrep Theory Pract, 19(3), 91-104.

Krueger, N.F. \& Carsrud, A.L. (1993). Entrepreneurial intentions: applying the theory of planned behavior. Entrep Region Dev, 5(4), 315-330. http:// dx.doi.org/ 10.1080/08985629300000020

Liñán, F \& Chen, Y. (2009). Development and cross-cultural application of a specific instrument to measure entrepreneurial intentions. Entrep Theory Pract, 33(3), 593-617. http://dx.doi. org/10.1111/j.1540-6520.2009.00318.x

Liñán, F., Urbano, D. \& Guerrero, M. (2011). Regional variations in entrepreneurial cognitions: Start-up intentions of university students in Spain. Entrep Region Dev, 23(3-4) http://dx.doi. org/10.1080/08985620903233929

Liñán, F., Battistelli, A. \& Moriano, J. (2008). Entrepreneurial intentions in Europe. En: Moriano, J.A., Gorgievski, M. \& Lukes, M. (Eds.), Teaching Psychology of Entrepreneurship. pp. 21-44.

Lucas, W.A., Cooper, S. Y., Ward, T. \& Cave, F. (2009). Industry placement, authentic experience and the development of venturing and technology self-efficacy. Technovation, 29(11), 738-752. http://doi.org/10.1016/j.technovation.2009.06.002

Mira-Solves, I. (2006). La creación de empresas por titulados universitarios. Una aproximación a los factores de influencia del ámbito de la Universidad". Tesis doctoral. Universidad Miguel Hernández. http://dspace.umh.es/bitstream/11000/752/1/Tesis_Ignacio_Mira.pdf

Peterman, N. E. \& Kennedy, J. (2003). Enterprise education: Influencing students' perceptions of entrepreneurship. Entrep Theory Pract, 28(2)129-144. http://doi.org/10.1046/j.1540-6520.2003.00035.x

Ripollés, M. (1995). Una propuesta de definición de entrepreneur. Eur J Manag Bus Econ, 3(3), 127136.

Ribeiro, D. (2003). Modeling the enterprising character of European firms. Eur Bus Rev, 15(1), 29-37. http://dx.doi.org/10.1108/09555340310455173

Robinson, P.B. \& Sexton, E.A. (1994). The effect of education and experience of self-employment success. J Bus Venturing, 9(2), 141-156. https://doi. org/10.1016/0883-9026(94)90006-X

Rodríguez-Gutiérrez, M.J \& Santos-Cumplido, F.J. (2008). La actividad emprendedora de las muje- res y el proceso de creación de empresas. ICE-Revista de Economía, 841,117-132. http://www. revistasice.com/cachepdffICE_841_117-132_67EE5F31803C91CF9CCD4A7A146116B6.pdf.

Rokearch, M. (1973). The nature of human values. Michigan University. Nueva York: Free Press (Eds.). 438p.

Rusque, A.M. (2004). Reflexiones en torno a un programa emprendedor para las universidades latinoamericanas. Anales de la Universidad Metropolitana, 4(1), 243-252.

Ruiz-Navarro, J., Rojas-Vázquez, A. \& Suárez-Llorents, A. (2008). Las actitudes de los estudiantes universitarios de Andalucía ante la creación de empresas. Universidad de Cadiz, España (Eds.). 100p. https://web.stanford.edu/group/ree/archives/ archive09/latinamerica/presentations/navarro-intenciones_emprendedoras.pdf.

Soto-Laguerre, L. M. (2006). Los mandamientos de un Emprendedor en busca del éxito. http://www. business-opportunities.biz/espanol/2005/09/17/ los-mandamientos-de-un-emprendedor-en-busca-del-exito/.

Shapero, A. \& Sokol, L. (1982). Social dimensions of entrepreneurship. In: Kent, C.A., Sexton, D.L., \& Vesper, K.H. (Eds.). Englewood Cliffs (NJ): Prentice Hall. pp. 72-90.

Thompson, P. (1986). Characteristics of the small business entrepreneur in Canada. J Small Bus Entrepreneurship, 4(3), 5 -11. http://dx.doi.org/10.108 0/08276331.1987.10600265

Urbano-Pulido, D. (2006). La creación de empresas en Catalunya: Organismos de apoyo y actitudes hacia la actividad emprendedora. Departament de Treball and Industry, Centre d'Tnnovació i Desenvolupament Empresarial- CIDEM (Eds.). 230p.

Varela, R. (2008). La Universidad y su función en el desarrollo empresarial latinoamericano. Revista Javeriana, 743, 32-43.

Vesper, K.H., \& William, B. \& Gartner (1997). Measuring progress in the entrepreneurship education. J Bus Venturing, 12(5), 403-421. https://doi. org/10.1016/S0883-9026 (97)00009-8

ZHAO, H., Siebert, S. E., \& Hills, G. E. (2005). The mediating role of self-efficacy in the development of entrepreneurial intentions. J Appl Psychol, 90(6), 1265-1272. http://dx.doi.org/ 10.1037/00219010.90 .6 .1265 